\title{
Riemann-Hurwitz formula in basic $\mathbb{Z}_{S}$-extensions
}

\author{
by
}

Yi Ouyang (Hefei and Minneapolis, Minn.) and FeI Xu (Hefei)

1. Introduction. Let $p$ be a prime number and $\mathbb{F}$ be a CM-field. Let $\mathbb{F}_{\infty}$ be the cyclotomic $\mathbb{Z}_{p}$-extension of $\mathbb{F}$. For every $n$, we have a unique subextension $\mathbb{F}_{n}$ of degree $p^{n}$ over $\mathbb{F}$ in $\mathbb{F}_{\infty}$. We denote by $\mathbb{F}^{+}$the maximal real subfield of $\mathbb{F}$, and let $h_{n}^{-}$be the relative class number of $\mathbb{F}_{n} / \mathbb{F}_{n}^{+}$. Then we have a well known result:

$$
\operatorname{ord}_{p}\left(h_{n}^{-}\right)=\mu^{-} p^{n}+\lambda^{-} n+\nu^{-},
$$

$\mu^{-} \geq 0, \lambda^{-} \geq 0$, and $\nu^{-}$are integers, when $n$ is sufficiently large.

Let $\mathbb{E}$ be a CM-field and a $p$-extension of $\mathbb{F}$. Under the assumption $\mu_{\mathbb{F}}^{-}=0$, Y. Kida ([5]) proved a striking analogue of the classical RiemannHurwitz genus formula from the theory of compact Riemann surfaces, by describing the behavior of $\lambda^{-}$in the $p$-extension. His result can be stated as follows:

Theorem 0 (see $\left[8\right.$, Theorem 4.1]). $\mu_{\mathbb{F}}^{-}=0$ if and only if $\mu_{\mathbb{E}}^{-}=0$, and when this is the case,

$$
\lambda_{\mathbb{E}}^{-}-\delta_{\mathbb{E}}=\left[\mathbb{E}_{\infty}: \mathbb{F}_{\infty}\right]\left(\lambda_{\mathbb{F}}^{-}-\delta_{\mathbb{F}}\right)+\sum_{\omega^{\prime}}\left(e\left(\omega^{\prime} / \nu^{\prime}\right)-1\right)-\sum_{\omega}(e(\omega / \nu)-1),
$$

where the summation is taken over all places $\omega^{\prime}$ on $\mathbb{E}_{\infty}\left(\right.$ resp. $\omega$ on $\left.\mathbb{E}_{\infty}^{+}\right)$ which do not lie above $p$ and $\nu^{\prime}=\left.\omega^{\prime}\right|_{\mathbb{F}_{\infty}}\left(\right.$ resp. $\left.\nu=\left.\omega\right|_{\mathbb{F}_{\infty}^{+}}\right), e(\omega / \nu)($ resp. $\left.e\left(\omega^{\prime} / \nu^{\prime}\right)\right)$ is the ramification index of $\omega$ (resp. $\left.\omega^{\prime}\right)$ over $\nu$ (resp. $\left.\nu^{\prime}\right)$ and $\delta_{\mathbb{E}}=1$ or $0\left(\right.$ resp. $\delta_{\mathbb{F}}=1$ or 0$)$ according as $\mathbb{E}$ (resp. $\left.\mathbb{F}\right)$ contains $\zeta_{p}\left(\right.$ or $\zeta_{4}$ if $p=2$ ) or not.

There are several ways to prove this result. K. Iwasawa ([4]) showed us a proof by using Galois cohomology. W. Sinnott ([8]) gave a proof by using the $p$-adic $L$-function and J. Satoh ([6]) obtained it by using the theory of $\Gamma$-transforms of rational functions. In this paper, we generalize the above result to basic $\mathbb{Z}_{S}$-extensions when $\mathbb{E}$ and $\mathbb{F}$ are abelian.

Let $S=\left\{p_{1}, \ldots, p_{s}\right\}$ be a finite set of primes, $\mathbb{Z}_{S}=\prod_{l \in S} \mathbb{Z}_{l}$ and $\mathbb{Q}_{S}$ be the $\mathbb{Z}_{S}$-extension of $\mathbb{Q}$. Then $\mathbb{F}_{S}=\mathbb{F} \mathbb{Q}_{S}$ is called the basic $\mathbb{Z}_{S}$-extension 
of $\mathbb{F}$. Let $N=p_{1}^{n_{1}} \ldots p_{s}^{n_{s}}$ and $\mathbb{F}_{N}$ be the unique subextension of degree $N$ of $\mathbb{F}_{S}$. Let $h_{N}^{-}$denote the relative class number of $\mathbb{F}_{N} / \mathbb{F}_{N}^{+}$. From a theorem of E. Friedman $([2])$, when $\mathbb{F}$ is an imaginary abelian number field, we have

$$
\operatorname{ord}_{p_{i}}\left(h_{N}^{-}\right)=\lambda^{-}\left(p_{i}, S\right) n_{i}+\nu^{-}\left(p_{i}, S\right)
$$

where all $n_{i}$ are sufficiently large and $p_{i} \in S$.

In this paper, using the relationship between $\lambda^{-}\left(p_{i}, S\right)$ and the $\lambda$-invariant of the Dirichlet character of $\mathbb{F}$, we obtain the following main result.

Theorem 1. For fixed $p \in S$, let $\mathbb{E}$ and $\mathbb{F}$ be imaginary abelian number fields and $\mathbb{E}$ be a p-extension of $\mathbb{F}$. We have

$$
\begin{aligned}
\lambda_{\mathbb{E}}^{-}(p, S)-\delta_{\mathbb{E}}= & {\left[\mathbb{E}_{S}: \mathbb{F}_{S}\right]\left(\lambda_{\mathbb{F}}^{-}(p, S)-\delta_{\mathbb{F}}\right) } \\
& +\sum_{\omega^{\prime}}\left(e\left(\omega^{\prime} / \nu^{\prime}\right)-1\right)-\sum_{\omega}(e(\omega / \nu)-1),
\end{aligned}
$$

where the summation is taken over all places $\omega^{\prime}$ on $\mathbb{E}_{S}$ (resp. $\omega$ on $\mathbb{E}_{S}^{+}$) which do not lie above $p$ and $\nu^{\prime}=\left.\omega^{\prime}\right|_{\mathbb{F}_{S}}\left(\right.$ resp. $\left.\nu=\left.\omega\right|_{\mathbb{F}_{S}^{+}}\right)$, and $e(\omega / \nu)($ resp. $\left.e\left(\omega^{\prime} / \nu^{\prime}\right)\right)$ is the ramification index of $\omega\left(\right.$ resp. $\left.\omega^{\prime}\right)$ over $\nu\left(\right.$ resp. $\left.\nu^{\prime}\right)$ and $\delta_{\mathbb{E}}=1$ or 0 (resp. $\delta_{\mathbb{F}}=1$ or 0$)$ according as $\mathbb{E}$ (resp. $\left.\mathbb{F}\right)$ contains $\zeta_{p}\left(\right.$ or $\zeta_{4}$ if $p=2$ ) or not.

2. Preliminaries. Let $p \in S$ be a fixed prime number and put

$$
q= \begin{cases}4, & p=2 \\ p, & p \neq 2 .\end{cases}
$$

Let $\omega_{p}$ be the Teichmüller character $\bmod q$. For every $m \in \mathbb{Z}$ with $(m, p)=1$ and $m \neq \pm 1$, we have

$$
m=\omega_{p}(m)\left(1+m_{1} p^{n_{m}}\right),
$$

with $m_{1} \in \mathbb{Z}_{p},\left(m_{1}, p\right)=1$ and $n_{m}$ being a positive integer. We let $\mathbb{Q}^{(p)}$ denote the basic $\mathbb{Z}_{p}$-extension on $\mathbb{Q}$ and $T=S-\{p\}$.

Let $\mathcal{O}$ be a ring of integers of a finite extension over $\mathbb{Q}_{p}$ and let $f(X)=$ $a_{0}+a_{1} X+\ldots \in \mathcal{O}[[X]]$ be a non-zero power series. We define

$$
\mu(f)=\min \left\{\operatorname{ord}_{p} a_{i}: i \geq 0\right\}, \quad \lambda(f)=\min \left\{i \geq 0: \operatorname{ord}_{p} a_{i}=\mu(f)\right\} .
$$

Clearly we have $\mu(f g)=\mu(f)+\mu(g)$ and $\lambda(f g)=\lambda(f)+\lambda(g)$ if $f, g$ are non-zero elements of $\mathcal{O}[[X]]$. So $\mu$ and $\lambda$ can be defined in the quotient field of $\mathcal{O}[[X]]$ in a natural way.

Let $\mathbb{Z}_{S}^{\times}$denote the unit group of $\mathbb{Z}_{S}$. So

$$
\mathbb{Z}_{S}^{\times}=U_{S} \times V_{S}
$$

where $V_{S}$ is the torsion part of $\mathbb{Z}_{S}^{\times}$and $U_{S}=\prod_{l \in S}\left(1+2 l \mathbb{Z}_{l}\right)$. Let \langle\rangle$_{S}$ and $\omega_{S}$ denote the projections from $\mathbb{Z}_{S}^{\times}$to $U_{S}$ and $V_{S}$ respectively. When $s=1, \omega_{S}$ is the Teichmüller character. Let $\theta$ be an odd primitive Dirichlet character 
with values in $\mathbb{C}_{p}$, where $\mathbb{C}_{p}$ is a fixed completion of the algebraic closure of $\mathbb{Q}_{p}$. Any primitive Dirichlet character whose conductor is divisible only by the primes in $S$ can be regarded as a character of $\mathbb{Z}_{S}^{\times}$. Such a character is called of the second kind for $S$ if it is trivial on $V_{S}$. For a character $\Psi$ of the second kind for $S$, we have the decomposition $\Psi=\Psi^{(p)} \Psi^{(T)}$, where $\Psi^{(p)}$ $\left(\operatorname{resp} . \Psi^{(T)}\right)$ is of the second kind for $p$ (resp. $T$ ) (see [9]).

Let $\theta$ be an odd primitive Dirichlet character with values in $\mathbb{C}_{p}$. Fix a generator $u$ of $U_{p}$. When $\theta \omega_{p}$ is not of the second kind for $p$, we define

$$
\lambda(\theta)=\lambda\left(g_{\theta}(X-1)\right),
$$

where

$$
g_{\theta}(X-1) \in 2 \mathcal{O}[[X-1]] \quad \text { with } \quad g_{\theta}\left(u^{s}-1\right)=L_{p}\left(s, \theta \omega_{p}\right)
$$

and $L_{p}\left(s, \theta \omega_{p}\right)$ is the $p$-adic $L$-function associated with $\theta \omega_{p}$. When $\theta \omega_{p}$ is of the second kind for $p$, we define $\lambda(\theta)=-1$. The following proposition is Theorem 1 of $[6]$.

Proposition 1. Let $\theta$ be an odd primitive Dirichlet character, $\tau$ be an even primitive Dirichlet character and $\mathcal{O}$ be the integer ring of the field generated over $\mathbb{Q}_{p}$ by the values of $\theta$ and $\tau$. Suppose

(1) $\tau$ has a p-power order and its conductor $l$ is a prime number,

(2) for all $a \in \mathbb{Z}, \theta \tau(a)=\theta(a) \tau(a)$.

Then

(i) if $\theta \neq \omega_{p}^{-1}$, we have

$$
\lambda(\theta \tau)= \begin{cases}\lambda(\theta)+p^{n_{l}} / q & \text { if } \theta(l) \equiv 1 \bmod \wp, \\ \lambda(\theta) & \text { if } \theta(l) \neq \equiv 1 \bmod \wp,\end{cases}
$$

where $\wp$ is a prime ideal of $\mathcal{O}$ above $p$,

(ii) if $\theta=\omega_{p}^{-1}$, we have

$$
\lambda(\theta \tau)=\frac{p^{n_{l}}}{q}-1
$$

Remark 1 . This proposition can also be proved by using the $p$-adic $L$-function (see $[8, \S 2])$.

Proposition 2. Let $\theta$ be an odd primitive Dirichlet character of order prime to $p, \tau$ be an even primitive Dirichlet character of p-power order and $\theta \tau(a)=\theta(a) \tau(a)$. Suppose the conductor $f(\tau)$ of $\tau$ is prime to $p$. Write $f(\tau)=\prod_{l} l^{k_{l}}$, where $k_{l} \geq 1$ and $l$ are primes. Then 
(i) $k_{l}=1$, for all $l$,

(ii) if $\theta \neq \omega_{p}^{-1}$, then

$$
\lambda(\theta \tau)=\lambda(\theta)+\sum_{\substack{l \\ \theta(l)=1}} \frac{p^{n_{l}}}{q},
$$

if $\theta=\omega_{p}^{-1}$, then

$$
\lambda(\theta \tau)=\left(\sum_{l} \frac{p^{n_{l}}}{q}\right)-1 .
$$

Proof. (i) By the Chinese Remainder Theorem, we have $\tau=\prod_{l} \tau_{l}$, where $l^{k_{l}}$ is the conductor of $\tau_{l}$ and $\tau_{l}$ has $p$-power order.

If $k_{l} \neq 1$, consider the natural map

$$
i: \mathbb{Z} /\left(l^{k_{l}}\right) \rightarrow \mathbb{Z} /\left(l^{k_{l}-1}\right) .
$$

For any $x \in \operatorname{ker} i, x$ has $l$-power order. Thus $\tau_{l}(x)$ is an $l$-power root of unity. Note $\tau_{l}$ has $p$-power order and $(p, l)=1$, and so we have $\tau_{l}(x)=1$. This is a contradiction because $l^{k_{l}}$ is the conductor of $\tau_{l}$.

(ii) When $\theta \neq \omega_{p}^{-1}$, the assertion follows from Proposition 1 and (i) since $\theta \tau(l) \equiv 1 \bmod \wp$ if and only if $\theta(l)=1$. If $\theta=\omega_{p}^{-1}$, then $l \equiv 1 \bmod p$ since $\tau_{l}$ has $p$-power order. Therefore $\theta(l) \equiv 1 \bmod \wp$ and we are done by Proposition 1.

3. The number of splitting primes. Let $\mathbf{k}$ be a finite abelian extension of $\mathbb{Q}$. In this section, we compute the number of primes of $\mathbf{k}_{S}$ above a prime number $l$, which is closely related to the characters of the Galois group. The character group of an abelian profinite group $G$ is the set of continuous homomorphisms from $G$ to the roots of unity in $\mathbb{C}_{p}^{\times}$with the induced topology. We denote this character group as $G^{\wedge}$.

Now we take $\chi \in \operatorname{Gal}\left(\mathbf{k}_{S} / \mathbb{Q}\right)^{\wedge}$. Then ker $\chi$ is a close subgroup with finite index of $\operatorname{Gal}\left(\mathbf{k}_{S} / \mathbb{Q}\right)$ (an open subgroup) and $\chi$ is essentially a usual Dirichlet character. Let $\mathbf{k}^{\chi}$ be the subfield of $\mathbf{k}_{S}$ fixed by ker $\chi$. Then we define

$$
\chi(l)= \begin{cases}0 & \text { if } l \text { is ramified in } \mathbf{k}^{\chi}, \\ \chi\left(\operatorname{Frob}_{l}\right) & \text { if } l \text { is unramified in } \mathbf{k}^{\chi} .\end{cases}
$$

Keeping the above notations, we have the following lemma:

Lemma 1. For any prime number $l$,

(i) there are finitely many primes in $\mathbf{k}_{S}$ above $l$,

(ii) the number of primes above $l$ in $\mathbf{k}_{S}$ is equal to

$$
\#\left\{\chi \in \operatorname{Gal}\left(\mathbf{k}_{S} / \mathbb{Q}\right)^{\wedge}: \chi(l)=1\right\} .
$$


Proof. (i) First consider $S=\{p\}$. Let $\mathcal{Q}$ be a prime in $\mathbf{k}$ above $l$. If $l=p$, the assertion is trivial by [10, Lemma 13.3]. If $l \neq p$, then $\mathcal{Q}$ is unramified in $\mathbf{k}_{S} / \mathbf{k}$. Write

$$
l=\omega_{p}(l)\left(1+p^{n_{l}} l_{1}\right) .
$$

Then the number of primes of $\mathbf{k}$ above $\mathcal{Q}$ is equal to

$$
\begin{aligned}
& \#\left(\operatorname{Gal}\left(\mathbf{k}_{S} / \mathbf{k}\right) / \overline{\left\langle\operatorname{Frob}_{\mathcal{Q}}\right\rangle}\right) \\
& \quad \leq \#\left(\operatorname{Gal}\left(\mathbb{Q}^{(p)} / \mathbb{Q}\right) / \overline{\left\langle\operatorname{Frob}_{l}\right\rangle}\right) \cdot[\mathbf{k}: \mathbb{Q}] \leq p^{n_{l}}[\mathbf{k}: \mathbb{Q}]<\infty
\end{aligned}
$$

and the case $s=1$ is proved.

If $s>1$, let $D(\mathcal{Q})$ be the decomposition group of $\mathcal{Q}$. Then $D(\mathcal{Q})$ is a closed subgroup of $\mathbb{Z}_{S}$ and has the form $p_{1}^{t_{1}} \mathbb{Z}_{p_{1}} \times \ldots \times p_{s}^{t_{s}} \mathbb{Z}_{p_{s}}, 0 \leq t_{i} \leq \infty, i=$ $1, \ldots, s$, where $p_{i}^{\infty} \mathbb{Z}_{p_{i}}=0$. It is sufficient to prove that $t_{i}<\infty, i=1, \ldots, s$. Suppose $t_{i}=\infty$. Let $\mathbf{k}^{\left(p_{i}\right)} \subseteq \mathbb{L}$ be a basic $\mathbb{Z}_{p_{i}}$-extension of $\mathbf{k}$ and $D^{\left(p_{i}\right)}(\mathcal{Q})$ be the decomposition group of $\mathcal{Q}$ over $\mathbf{k}^{\left(p_{i}\right)}$. So we have

$$
D^{\left(p_{i}\right)}(\mathcal{Q})=\left.D(\mathcal{Q})\right|_{\operatorname{Gal}\left(\mathbf{k}^{\left(p_{i}\right)} / \mathbf{k}\right)}=0 .
$$

This is a contradiction to the case of $s=1$ and (i) is proved.

(ii) Let $D(l)$ denote the decomposition group of a prime in $\mathbf{k}_{S}$ above $l$. Then the number of primes in $\mathbf{k}_{S}$ above $l$ is equal to

$$
\begin{aligned}
\#\left(\operatorname{Gal}\left(\mathbf{k}_{S} / \mathbb{Q}\right) / D(l)\right) & =\#\left(\left(\operatorname{Gal}\left(\mathbf{k}_{S} / \mathbb{Q}\right) / D(l)\right)^{\wedge}\right) \\
& =\#\left\{\chi \in \operatorname{Gal}\left(\mathbf{k}_{S} / \mathbb{Q}\right)^{\wedge}: \chi(l)=1\right\} .
\end{aligned}
$$

This is the desired result.

Remark 2. Lemma 1 is not true for arbitrary $\mathbb{Z}_{S}$-extension (see $[10$, Ex. 13.2]).

From Lemma 1, we immediately have the following lemma:

Lemma 2. Suppose $\mathbf{k} \cap \mathbb{Q}_{S}=\mathbb{Q}, p \in S$ with $p \nmid[\mathbf{k}: \mathbb{Q}], T=S-\{p\}$ and $l$ is a prime number different from $p$. Then the number of prime ideals above $l$ in $\mathbf{k} \mathbb{Q}_{S}$ is

$$
\begin{aligned}
\#\left\{\chi \in \operatorname{Gal}\left(\mathbf{k} \mathbb{Q}_{T} / \mathbb{Q}\right)^{\wedge}: \chi(l)=1\right\} \#\left\{\chi \in \operatorname{Gal}\left(\mathbb{Q}^{(p)} / \mathbb{Q}\right)^{\wedge}: \chi(l)=1\right\} \\
=\left(p^{n_{l}} / q\right) \#\left\{\chi \in \operatorname{Gal}\left(\mathbf{k} \mathbb{Q}_{T} / \mathbb{Q}\right)^{\wedge}: \chi(l)=1\right\} .
\end{aligned}
$$

Proof. By Lemma 1, it is sufficient to prove

$$
\begin{aligned}
\#\left\{\chi \in \operatorname{Gal}\left(\mathbf{k} \mathbb{Q}_{S} / \mathbb{Q}\right)^{\wedge}: \chi(l)=1\right\} \\
\quad=\#\left\{\chi \in \operatorname{Gal}\left(\mathbf{k} \mathbb{Q}_{T} / \mathbb{Q}\right)^{\wedge}: \chi(l)=1\right\} \#\left\{\chi \in \operatorname{Gal}\left(\mathbb{Q}^{(p)} / \mathbb{Q}\right)^{\wedge}: \chi(l)=1\right\} .
\end{aligned}
$$

Since

we have

$$
\operatorname{Gal}\left(\mathbf{k} \mathbb{Q}_{S} / \mathbb{Q}\right) \cong \operatorname{Gal}\left(\mathbf{k} \mathbb{Q}_{T} / \mathbb{Q}\right) \times \operatorname{Gal}\left(\mathbb{Q}^{(p)} / \mathbb{Q}\right),
$$

$$
\operatorname{Gal}\left(\mathbf{k} \mathbb{Q}_{S} / \mathbb{Q}\right)^{\wedge} \cong \operatorname{Gal}\left(\mathbf{k} \mathbb{Q}_{T} / \mathbb{Q}\right)^{\wedge} \times \operatorname{Gal}\left(\mathbb{Q}^{(p)} / \mathbb{Q}\right)^{\wedge}
$$


Therefore for any $\chi \in \operatorname{Gal}\left(\mathbf{k} \mathbb{Q}_{S} / \mathbb{Q}\right)^{\wedge}$, we have $\chi=\chi_{T} \cdot \chi_{p}$, with $\chi_{T} \in$ $\operatorname{Gal}\left(\mathbf{k} \mathbb{Q}_{T} / \mathbb{Q}\right)^{\wedge}, \chi_{p} \in \operatorname{Gal}\left(\mathbb{Q}^{(p)} / \mathbb{Q}\right)^{\wedge}$ and $\chi(l)=\chi_{T}(l) \chi_{p}(l)$. Note $\chi_{p}(l)$ is a $p$-power root of unity and $\chi_{T}(l)$ is not, so we have

$$
\chi(l)=1 \Leftrightarrow \chi_{T}(l)=1 \text { and } \chi_{p}(l)=1
$$

and Lemma 2 is proved.

4. Proof of Theorem 1. First let $\mathbf{k}$ be a finite abelian extension of $\mathbb{Q}$ and we use the following notations associated with $\mathbf{k}$ :

- $X_{\mathbf{k}}\left(\right.$ resp. $\left.X_{\mathbf{k}}^{-}\right)$: the set of all (resp. odd) Dirichlet characters associated with $\mathbf{k}$

- $X_{\mathbf{k}}(l)\left(\right.$ resp. $\left.X_{\mathbf{k}}^{-}(l)\right)$ : all the elements of $X_{\mathbf{k}}\left(\right.$ resp. $\left.X_{\mathbf{k}}^{-}\right)$whose conductors are divisible by a prime number $l$.

- $J_{\mathbf{k}}(l)$ : all the elements of $X_{\mathbf{k}}$ whose conductors are prime to a prime number $l$.

- We write $\chi_{\mathbf{k}}$ for an element of $X_{\mathbf{k}}$ and $\mathfrak{f}_{\mathbf{k}}$ as the conductor of $\mathbf{k}$. Let $e, f$ and $g$ denote the usual meaning as the ramification index, the residue class degree, the number of splitting primes respectively. For a prime number $l$, by $[10$, Th. 3.7], we have

$$
\# J_{\mathbf{k}}(l)=f_{\mathbf{k}}(l) g_{\mathbf{k}}(l) \text { and } \#\left(X_{\mathbf{k}} / J_{\mathbf{k}}(l)\right)=e_{\mathbf{k}}(l) .
$$

Now $\mathbb{E}, \mathbb{F}$ are the same as in Section 1 . Let $\mathbb{K}$ be the maximal $p$-extension of $\mathbb{Q}$ in $\mathbb{E}$, and $\mathbb{L}$ be the maximal extension of $\mathbb{Q}$ in $\mathbb{E}$ with $p \nmid[\mathbb{L}: \mathbb{Q}]$. $\omega\left(\right.$ resp. $\left.\omega^{\prime}\right)$ is a prime of $\mathbb{E}_{S}^{+}$(resp. $\mathbb{E}_{S}$ ) which does not lie over the prime $p, \nu=\left.\omega\right|_{\mathbb{F}_{S}^{+}}\left(\right.$resp. $\left.\nu^{\prime}=\omega^{\prime}{\mid \mathbb{F}_{S}}\right)$ and $u=\left.\omega\right|_{\mathbb{L}_{S}^{+}}\left(\right.$resp. $\left.u^{\prime}=\left.\omega\right|_{\mathbb{L}_{S}}\right)$.

Suppose $\left.\omega\right|_{\mathbb{Q}}=l \neq p$. Since the residue field at $u$ or $u^{\prime}$ has no finite $p$-extensions, it is clear that $f(\omega / u)=f\left(\omega^{\prime} / u^{\prime}\right)=1$. Furthermore,

$$
\begin{aligned}
& e_{\mathbb{K}}(l)=e\left(\omega^{\prime} / u^{\prime}\right), e_{\mathbb{K}^{+}}(l)=e(\omega / u), \\
& \# J_{\mathbb{K}}=g\left(\omega^{\prime} / u^{\prime}\right), \quad \# J_{\mathbb{K}^{+}}=g(\omega / u) .
\end{aligned}
$$

We also note the following:

1) It is easy to check that if Theorem 1 holds for two of $\mathbb{E} / \mathbb{F}, \mathbb{K} / \mathbb{F}$ and $\mathbb{E} / \mathbb{K}$, it holds for the third. This allows us to reduce ourselves to the case where $[\mathbb{F}: \mathbb{Q}]$ is not divisible by $p$ for $p>2$.

2) We can also assume $\mathbb{E} \cap \mathbb{F}_{S}=\mathbb{F}, \mathbb{F} \cap \mathbb{Q}_{S}=\mathbb{Q}$ and the conductor of $\mathbb{E}$ is not divisible by $q p$, since any number field between $\mathbb{E}$ and $\mathbb{E}_{S}$ has the same $\lambda(p, S)$-invariant as that of $\mathbb{E}$.

3) By the above assumption, we have $\left[\mathbb{E}_{S}: \mathbb{F}_{S}\right]=[\mathbb{E}: \mathbb{F}]$ and $\mathbb{E} \cap \mathbb{Q}_{S}=\mathbb{Q}$.

With the above notations, we have the following lemma: 


\section{LEMMA 3.}

$$
\begin{aligned}
& \sum_{\omega^{\prime}}\left(e\left(\omega^{\prime} / \nu^{\prime}\right)-1\right)-\sum_{\omega}(e(\omega / \nu)-1) \\
& \quad=\left\{\begin{array}{lr}
\sum_{l} p^{n_{l}-1} \# X_{\mathbb{K}}(l) \#\left\{\chi_{\mathbb{F}} \Psi^{(T)}: \chi_{\mathbb{F}} \text { odd }, \chi_{\mathbb{F}} \Psi^{(T)}(l)=1\right\} & \text { if } p>2, \\
\sum_{l} 2^{n_{l}-2}\left\{\# X_{\mathbb{K}}^{-}(l)-[\mathbb{E}: \mathbb{F}] \# X_{\mathbb{F} \cap \mathbb{K}}(l)^{-}\right\} \#\left\{\chi_{\mathbb{L}} \Psi^{(T)}: \chi_{\mathbb{L}} \Psi^{(T)}(l)=1\right\}, & \text { if } p=2,
\end{array}\right.
\end{aligned}
$$

where $\omega^{\prime}$ (resp. $\left.\omega\right)$ runs over all the primes in $\mathbb{E}_{S}\left(\right.$ resp. $\left.\mathbb{E}_{S}^{+}\right)$which do not lie over $p, l$ runs over all the prime numbers different from $p$ and $\Psi^{(T)}$ is taken over the characters of $\operatorname{Gal}\left(\mathbb{Q}_{T} / \mathbb{Q}\right)$.

Proof. We have

$$
\begin{aligned}
\sum_{\omega^{\prime}}\left(e\left(\omega^{\prime} / u^{\prime}\right)\right. & -1)-\sum_{\omega}(e(\omega / u)-1) \\
= & \sum_{u^{\prime}} g\left(\omega^{\prime} / u^{\prime}\right)\left(e\left(\omega^{\prime} / u^{\prime}\right)-1\right)-\sum_{u} g(\omega / u)(e(\omega / u)-1) .
\end{aligned}
$$

If $p>2$, then $\mathbb{F}=\mathbb{L}, \nu=u, \nu^{\prime}=u^{\prime}$ and $\mathbb{K}=\mathbb{K}^{+}$. By Lemma 2, we have

$$
\begin{aligned}
(*)= & \sum_{l \neq p} \# X_{\mathbb{K}}(l) \sum_{u^{\prime} \cap \mathbb{Q}=l} 1-\sum_{l \neq p} \# X_{\mathbb{K}}(l) \sum_{u \cap \mathbb{Q}=l} 1 \\
= & \sum_{l \neq p} \# X_{\mathbb{K}}(l) \#\left\{\chi_{\mathbb{F}} \Psi^{(T)}: \chi_{\mathbb{F}} \Psi^{(T)}(l)=1\right\} p^{n_{l}-1} \\
& -\sum_{l \neq p} \# X_{\mathbb{K}}(l) \#\left\{\chi_{\mathbb{F}^{+}} \Psi^{(T)}: \chi_{\mathbb{F}} \Psi^{(T)}(l)=1\right\} p^{n_{l}-1} \\
= & \sum_{l \neq p} \# X_{\mathbb{K}}(l) p^{n_{l}-1} \#\left\{\chi_{\mathbb{F}} \Psi^{(T)}: \chi_{\mathbb{F}} \text { odd, } \chi_{\mathbb{F}} \Psi^{(T)}(l)=1\right\} .
\end{aligned}
$$

For $p=2$, we have $\mathbb{F} \supset \mathbb{L}$ and $\mathbb{L}=\mathbb{L}^{+}$. So

$$
\begin{aligned}
(*) & =\sum_{l \neq p} \# X_{\mathbb{K}}(l) \sum_{\left.u^{\prime}\right|_{\mathbb{Q}}=l} 1-\sum_{l \neq p} \# X_{\mathbb{K}^{+}}(l) \sum_{\left.u\right|_{\mathbb{Q}}=l} 1 \\
& =\sum_{l \neq p} \# X_{\mathbb{K}}^{-}(l) 2^{n_{l}-2} \#\left\{\chi_{\mathbb{L}} \Psi^{(T)}: \chi_{\mathbb{L}} \Psi^{(T)}(l)=1\right\} .
\end{aligned}
$$

Let $\mathbb{E}=\mathbb{F}$. We have

(2)

$$
\begin{aligned}
\sum_{\nu^{\prime}}\left(e\left(\nu^{\prime} / u^{\prime}\right)-1\right) & -\sum_{\nu}(e(\nu / u)-1) \\
& =\sum_{l \neq p} 2^{n_{l}-2} \# X_{\mathbb{K} \cap \mathbb{F}}^{-}(l) \#\left\{\chi_{\mathbb{L}} \Psi^{(T)}: \chi_{\mathbb{L}} \Psi^{(T)}(l)=1\right\} .
\end{aligned}
$$

Since $\left[\mathbb{E}_{S}: \mathbb{F}_{S}\right]=[\mathbb{E}: \mathbb{F}]$ and $f\left(\omega^{\prime} / \nu^{\prime}\right)=1$, we have 


$$
e\left(\omega^{\prime} / \nu^{\prime}\right) g\left(\omega^{\prime} / \nu^{\prime}\right)=[\mathbb{E}: \mathbb{F}], \quad e\left(\omega^{\prime} / u^{\prime}\right)=e\left(\omega^{\prime} / \nu^{\prime}\right) e\left(\nu^{\prime} / u^{\prime}\right) .
$$

Then

$$
\begin{aligned}
{[\mathbb{E}: \mathbb{F}] } & \sum_{\nu^{\prime}}\left(e\left(\nu^{\prime} / u^{\prime}\right)-1\right) \\
& =\sum_{\nu^{\prime}} g\left(\omega^{\prime} / \nu^{\prime}\right)\left(e\left(\omega^{\prime} / u^{\prime}\right)-e\left(\omega^{\prime} / \nu^{\prime}\right)\right)=\sum_{\omega^{\prime}}\left(e\left(\omega^{\prime} / u^{\prime}\right)-e\left(\omega^{\prime} / \nu^{\prime}\right)\right) .
\end{aligned}
$$

The same is true for $\omega, u, \nu$. By (1) and (2), we obtain

$$
\begin{aligned}
\sum_{\omega^{\prime}} & \left(e\left(\omega^{\prime} / \nu^{\prime}\right)-1\right)-\sum_{\omega}(e(\omega / \nu)-1) \\
& =\sum_{l \neq p} 2^{n_{l}-2}\left\{\# X_{\mathbb{K}}^{-}(l)-[\mathbb{E}: \mathbb{F}] \# X_{\mathbb{K} \cap \mathbb{F}}^{-}(l)\right\} \#\left\{\chi_{\mathbb{L}} \Psi^{(T)}: \chi_{\mathbb{L}} \Psi^{(T)}(l)=1\right\} .
\end{aligned}
$$

Now we begin our proof of the main theorem.

Proof of Theorem 1 . We know that for any imaginary abelian field $\mathbf{k}, \lambda(p, S)$ satisfies the following relation (cf. [9]):

$$
\lambda_{\mathbf{k}}^{-}(p, S)=\delta_{\mathbf{k}}+\sum_{\theta} \sum_{\Psi^{(T)}} \lambda\left(\theta \Psi^{(T)}\right),
$$

where the outer sum is taken over all odd characters of $\mathbf{k} / \mathbb{Q}$ and the inner sum is taken over all $\Psi^{(T)} \in \operatorname{Gal}\left(\mathbb{Q}_{T} / \mathbb{Q}\right)^{\wedge}$ with $\lambda\left(\theta \Psi^{(T)}\right) \neq 0$, and $\delta_{\mathbf{k}}=1$ if and only if $\omega_{p}$ is a character of $\mathbf{k} / \mathbb{Q}$. Therefore

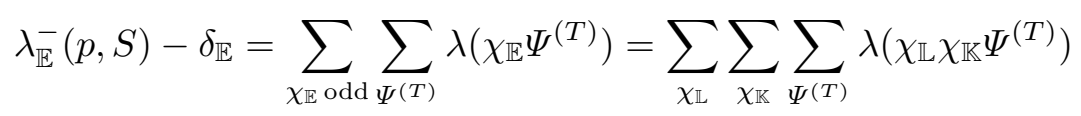

where $\chi_{\mathbb{K}} \chi_{\mathbb{L}}$ is odd.

When $p>2$, the conductor of $\chi \in \operatorname{Gal}(\mathbb{K} / \mathbb{Q})^{\wedge}$ is not divisible by $p$ since $\mathfrak{f}_{\mathbb{E}}$ is a not divisible by $p^{2}$ and $[\mathbb{K}: \mathbb{Q}]$ is a $p$-power. Note $\mathbb{L}=\mathbb{F}$ and $\mathbb{K}=\mathbb{K}^{+}$ in this case. By Propositions 1 and 2, we have

$$
\begin{aligned}
(* *) & =\sum_{\chi_{\mathbb{F}} \text { odd }} \sum_{\chi_{\mathbb{K}}} \sum_{\Psi^{(T)}}\left(\lambda\left(\chi_{\mathbb{F}} \Psi^{(T)}\right)+\sum_{\substack{l \mid f\left(\chi_{\mathbb{K}}\right) \\
\chi_{\mathbb{F}} \Psi^{(T)}(l)=1}} p^{n_{l}-1}\right) \\
& =[\mathbb{E}: \mathbb{F}] \sum_{\chi_{\mathbb{F}} \text { odd } \Psi^{(T)}} \lambda\left(\chi_{\mathbb{F}} \Psi^{(T)}\right)+\sum_{\chi_{\mathbb{F}} \text { odd } \Psi^{(T)}} \sum_{\substack{l \neq p \\
\chi_{\mathbb{F}} \Psi^{(T)}(l)=1}} \# X_{\mathbb{K}}(l) p^{n_{l}-1} \\
& =[\mathbb{E}: \mathbb{F}]\left(\lambda_{\mathbb{F}}^{-}(p, S)-\delta_{\mathbb{F}}\right)+\sum_{l \neq p} p^{n_{l}-1} \# X_{\mathbb{K}}(l) \sum_{\chi_{\mathbb{F}} \text { odd }, \chi_{\mathbb{F}} \Psi^{(T)}(l)=1} 1 \\
& =[\mathbb{E}: \mathbb{F}]\left(\lambda_{\mathbb{F}}^{-}(p, S)-\delta_{\mathbb{F}}\right)
\end{aligned}
$$




$$
\begin{aligned}
& +\sum_{l \neq p} p^{n_{l}-1} \# X_{\mathbb{K}}(l) \#\left\{\chi_{\mathbb{F}} \Psi^{(T)}: \chi_{\mathbb{F}} \text { odd }, \chi_{\mathbb{F}} \Psi^{(T)}(l)=1\right\} \\
= & {[\mathbb{E}: \mathbb{F}]\left(\lambda_{\mathbb{F}}^{-}(p, S)-\delta_{\mathbb{F}}\right)+\sum_{\omega^{\prime}}\left(e\left(\omega^{\prime} / \nu^{\prime}\right)-1\right)-\sum_{\omega}(e(\omega / \nu)-1) . }
\end{aligned}
$$

If $p=2$, then $\mathbb{L}=\mathbb{L}^{+}, \mathbb{L} \subset \mathbb{F}$ and the conductor of each character of $\mathbb{K}$ is not divisible by 8 . By [6, Th. 1$]$,

$$
\sum_{\chi_{\mathbb{K}} \text { odd }} \lambda\left(\chi_{\mathbb{K}}\right)=\sum_{l \neq p} 2^{n_{l}-2} \# X_{\mathbb{K}}^{-}(l)-\left[\mathbb{K}^{+}: \mathbb{Q}\right] .
$$

Since $\mathbb{K} \cap \mathbb{F}$ is an imaginary abelian extension of $\mathbb{Q}$, we can choose a primitive odd character $\chi_{0}$ of $\operatorname{Gal}((\mathbb{F} \cap \mathbb{K}) / \mathbb{Q})$ with order 2 . Then, for any $\chi \in X_{\mathbb{K}}^{-}$, we have $\chi=\chi_{0} \tilde{\chi}$ with $\tilde{\chi} \in X_{\mathbb{K}^{+}}$. By Propositions 1 and 2, we have

$$
\begin{aligned}
\sum_{\chi_{\mathbb{K}} \text { odd }} \sum_{\chi_{\mathbb{L}} \Psi^{(T)} \neq 1} \lambda\left(\chi_{\mathbb{K}} \chi_{\mathbb{L}} \Psi^{(T)}\right) & \\
= & \sum_{l \neq p} 2^{n_{l}-2} \# X_{\mathbb{K}}^{-}(l) \#\left\{\chi_{\mathbb{L}} \Psi^{(T)} \neq 1: \chi_{\mathbb{L}} \Psi^{(T)}(l)=1\right\} \\
& +\left[\mathbb{K}^{+}: \mathbb{Q}\right] \sum_{\chi_{\mathbb{L}} \Psi^{(T)} \neq 1} \lambda\left(\chi_{0} \chi_{\mathbb{L}} \Psi^{(T)}\right) .
\end{aligned}
$$

Therefore

$$
\begin{aligned}
(* *)= & \sum_{\chi_{\mathbb{L}}} \sum_{\chi_{\mathbb{K}} \text { odd }} \sum_{\Psi^{(T)}} \lambda\left(\chi_{\mathbb{K}} \chi_{\mathbb{L}} \Psi^{(T)}\right) \\
= & \sum_{\chi_{\mathbb{K}} \text { odd }} \sum_{\chi_{\mathbb{L}} \Psi^{(T)} \neq 1} \lambda\left(\chi_{\mathbb{K}} \chi_{\mathbb{L}} \Psi^{(T)}\right)+\sum_{\chi_{\mathbb{K}} \text { odd }} \lambda\left(\chi_{\mathbb{K}}\right) \\
= & {\left[\mathbb{K}^{+}: \mathbb{Q}\right]\left(\sum_{\chi_{\mathbb{L}} \Psi^{(T)} \neq 1} \lambda\left(\chi_{0} \chi_{\mathbb{L}} \Psi^{(T)}\right)-1\right) } \\
& +\sum_{l \neq p} 2^{n_{l}-2} \# X_{\mathbb{K}}^{-}(l) \#\left\{\chi_{\mathbb{L}} \Psi^{(T)}: \chi_{\mathbb{L}} \Psi^{(T)}(l)=1\right\} .
\end{aligned}
$$

If we set $\mathbb{E}=\mathbb{F}$ in the above equality, then we obtain

(4) $\lambda_{\mathbb{F}}^{-}(2, S)-\delta_{\mathbb{F}}=\left[\mathbb{K}^{+} \cap \mathbb{F}: \mathbb{Q}\right]\left(\sum_{\chi_{\mathbb{L}} \Psi^{(T)} \neq 1} \lambda\left(\chi_{0} \chi_{\mathbb{L}} \Psi^{(T)}\right)-1\right)$

$$
+\sum_{l \neq p} 2^{n_{l}-2} \# X_{\mathbb{K} \cap \mathbb{F}}^{-}(l) \#\left\{\chi_{\mathbb{L}} \Psi^{(T)}: \chi_{\mathbb{L}} \Psi^{(T)}(l)=1\right\} .
$$

By $(3)-\left[\mathbb{E}^{+}: \mathbb{F}^{+}\right](4)$ we obtain the desired result since $\left[\mathbb{E}^{+}: \mathbb{F}^{+}\right]\left[\mathbb{K}^{+} \cap \mathbb{F}: \mathbb{Q}\right]$ $=\left[\mathbb{K}^{+}: \mathbb{Q}\right]$ and $[\mathbb{E}: \mathbb{F}]=\left[\mathbb{E}^{+}: \mathbb{F}^{+}\right]=\left[\mathbb{E}_{S}: \mathbb{F}_{S}\right]$.

Acknowledgements. Both authors would like to thank the referee for pointing out some mistakes and misprints both in English and in math- 
ematics. The second author was supported by Alexander von Humboldt Foundation and National Natural Science Foundation of China.

\section{References}

[1] N. Childress, $\lambda$-invariants and $\Gamma$-transforms, Manuscripta Math. 64 (1989), 359-375.

[2] E. Friedman, Ideal class groups in basic $\mathbb{Z}_{p_{1}} \times \ldots \times \mathbb{Z}_{p_{s}}$ extensions of abelian number fields, Invent. Math. 65 (1982), 425-440.

[3] K. Iwasawa, On $\Gamma$-extensions of algebraic number fields, Bull. Amer. Math. Soc. 65 (1959), 183-226.

[4] -, Riemann-Hurwitz formula and p-adic Galois representations for number fields, Tohôku Math. J. (2) 33 (1981), 263-288.

[5] Y. Kida, l-extensions of CM-fields and cyclotomic invariants, J. Number Theory 2 (1980), 519-528.

[6] J. Satoh, The Iwasawa $\lambda_{p}$-invariants of $\Gamma$-transforms of the generating functions of the Bernoulli numbers, Japan. J. Math. 17 (1991), 165-174 .

[7] W. Sinnott, On the $\mu$-invariant of the $\Gamma$-transform of a rational function, Invent. Math. 75 (1984), 273-282.

[8] -, On the p-adic L-functions and the Riemann-Hurwitz genus formula, Compositio Math. 53 (1984), 3-17.

[9] -,$\Gamma$-transforms of rational function measures on $\mathbb{Z}_{S}$, Invent. Math. 89 (1987), 139-157.

[10] L. C. Washington, Introduction to Cyclotomic Fields, Grad. Texts in Math. 83, Springer, 1982.

Department of Mathematics

University of Science and Technology of China

Hefei, Anhui 230026

People's Republic of China

E-mail: xulm@sunlxob.nsc.ustc.edu.cn
Current address:

School of Mathematics University of Minnesota Minneapolis, Minnesota 55455

U.S.A.

E-mail: youyang@math.umn.edu 